\title{
The concept of "Businesswoman" in French linguaculture and media discourse
}

\section{[Le concept de "femme d'affaires" dans la linguaculture et le discours médiatique français]}

\author{
Raigul M. Baktiyarova - Kalbike U. Yessenova - Marina R. Zheltukhina - \\ Irina V. Privalova - Elena B. Ponomarenko
}

DOI: 10.18355/XL.2021.14.04.07

\begin{abstract}
This article analyses linguistic, cultural, and cognitive aspects of the image "businesswoman" in the French language. The target of this article is to study the features of the concept "business woman" in French media discourse and the representation of this concept in French linguistic culture. The linguistic units that verbalize the concept "businesswoman" in French media discourse are described. The representative and axiological characteristics of the concept of businesswoman in the French language are defined. The article presents a multi-faceted analysis of the concept "businesswoman". The main modes of verbal exteriorization of the concept "businesswoman" with the help of the French language means are studied. The most important cognitive stereotypes are identified. These cognitive stereotypes serve as the reproduction base for the analyzed concept in the French language. It has been proved that the structures of the verbal exteriorization of the concept reflect the main model of a businesswoman in French cultural linguistics.
\end{abstract}

Key words: businesswoman, linguistic-cultural concept, linguistic awareness, world image, concept sphere, cognitive stereotype, media discourse

\section{Résumé}

Cet article analyse les aspects linguistiques, culturels et cognitifs de l'image de "femme d'affaires" en langue française. Le but de l'article est d'étudier les caractéristiques du concept de "femme d'affaires" dans le discours médiatique français et la représentation de ce concept dans la culture linguistique française. Les unités linguistiques qui verbalisent le concept de "femme d'affaires" dans le discours médiatique français sont décrites. Les caractéristiques représentatives et axiologiques du concept de "femme d'affaires" en langue française y sont définies. L'article présente une analyse multiforme du concept de "femme d'affaires". Les principaux modes d'objectivation linguistique du concept "femme d'affaires" dans l'imaginaire de la langue française y sont étudiés. Les stéréotypes cognitifs les plus importants sont identifiés. Ces stéréotypes cognitifs servent de base à la reproduction des concepts adoptés en français. Il a été prouvé que les structures de l'extériorisation verbale du concept reflètent le modèle principal d'une femme d'affaires en linguistique culturelle française.

Mots clés : femme d'affaires, concept linguistique-culturel, conscience linguistique, image du monde, conceptosphère, stéréotype cognitif, discours médiatique

\section{Introduction}

Le but de cet article est une étude approfondie du concept de "femme d'affaires", ou plutôt, comment ce concept fonctionne dans le discours médiatique français et comment il est représenté dans la culture linguistique française. Il est bien connu que le concept est une catégorie primordiale dans le domaine de la linguistique cognitive. 
À cet égard, les principales questions récemment examinées dans de nombreux travaux scientifiques concernent la définition et la signification du concept. La base de la description, de la recherche et de l'analyse du concept est la définition des approches et des méthodes. Par conséquent, quelle que soit l'ampleur et l'intégration de l'étude de ce complexe de questions, il n'a pas été possible jusqu'à présent d'étayer une compréhension unifiée de la catégorie du concept. Nous pensons que la méthodologie de représentation des concepts est une question tout à fait légitime, qui était et est toujours ouverte. Dans la littérature scientifique consacrée à l'étude du concept, il n'existe pas d'opinion unique et universellement reconnue concernant sa structure, son essence, sa nature linguistique, sa classification et sa différenciation.

La formation de la linguistique cognitive française s'est faite grâce aux recherches de G. Guillaume (Rastier, 1993). Le développement ultérieur de la théorie de la psychosystématique a été représenté par les concepts suivants : la théorie des opérations prédicatives (Culioli, 1999) et la théorie de la polyphonie (Ducrot, 1998). C. Fuchs estime qu'aujourd'hui toute recherche en linguistique est cognitive.

La linguistique cognitive moderne du texte vise à établir des correspondances entre représentations textuelles et méta-linguistiques, et représentations textuelles et conceptuelles (Fuchs, 2008).

G. Lazard (2007) ne reconnaît pas l'efficacité de la direction cognitive, selon lui, il convient de l'accepter comme linguistique traditionnelle (Lazard, 2007 : 14), car le point de départ de cette théorie est l'intuition (Lazard, $2007: 18$ ).

Cependant, parmi les nombreux courants de la linguistique française contemporaine, on peut trouver des travaux consacrés à des études cognitives spécifiques et à leurs auteurs : problèmes du modèle du français basé sur des mots auxiliaires au sens spatial (Borillo, 2006), problèmes de la grammaire applicative (Desclés, 1994); travaux consacrés aux problèmes de logique et de référence (Kleiber, 1981; Borillo, 2006; Lazard, 2007).

Trois directions principales dans la formation du concept incluent :

1) les unités lexicales qui créent une image du monde chez les représentants d'une langue donnée et forment l'essence de la conscience linguistique nationale. La conceptosphère de toute langue est représentée par un certain ensemble de concepts. Et ici, le plus important est la conceptualisation d'une image du monde. C'est-à-dire que toute unité lexicale peut être trouvée dans un certain nombre de concepts, capables de donner une expression sémantique dans un sens ;

2) les structures sémantiques, caractérisées par des traits linguistiques-culturels et définissant les représentants d'une ethnoculture donnée. Ces concepts ne peuvent pas former la conceptosphère, mais en montrent une certaine partie : la sphère conceptuelle ;

3) seules les structures sémantiques de base sont nécessaires pour définir la mentalité d'un peuple.

L'origine et la formation d'un nouveau «type » de femmes dans la société française les femmes d'affaires, sont directement liées à des facteurs extralinguistiques : les transformations sociopolitiques, historiques, économiques qui ont eu lieu au début du vingtième siècle. Comme nous le savons, l'assouplissement des restrictions liées au genre sur les facteurs extra-linguistiques a conduit à ce qu'un groupe de femmes d'affaires devienne l'objet d'étude. Ce phénomène socioculturel nouvellement apparu dans la vie de la société présente un intérêt de recherche considérable pour un très large éventail de domaines linguistiques. L'image de femme d'affaires dans la société française s'est développée parallèlement à la formation du nouveau système politicoéconomique. Nous pensons que les notions de business lady et business woman sont des synonymes absolus du concept de femme d'affaires; elles ont commencé à être

XLinguae, Volume 14 Issue 4, October 2021, ISSN 1337-8384, eISSN 2453-711X 
utilisées en russe dans les années 1990. Le concept de femme d'affaires est un calque sémantique des unités linguistiques susmentionnées de la langue anglaise.

La pertinence de cette recherche est déterminée par la priorité de la direction anthropologique dans la linguistique moderne et se caractérise par le fait que son objet central est une personne, une société, une image linguistique et conceptuelle du monde. En général, le concept de femme d'affaires est l'une des unités les plus significatives sur le plan social et culturel de la sphère conceptuelle des affaires. Après tout, la participation active des femmes dans les affaires est devenue un phénomène permanent dans la société moderne.

À l'heure actuelle, la conscience publique est façonnée par les médias qui sont la principale source de connaissance sur le concept de femme d'affaires. La lexicographie française n'enregistre pas la définition du concept spécifique de femme d'affaires, c'est-à-dire que le nom de ce concept n'est donné dans aucun dictionnaire, qu'il n'a pas été étudié dans le contexte linguistique.

Nous pensons que le concept de femme d'affaires doit être considéré dans l'aspect cognitif de la linguaculture française. Grâce à l'analyse du cadre cognitif, il est possible d'identifier les principaux critères cognitifs et lexico-sémantiques du discours médiatique.

\section{Révision littéraire}

Chaque langue classifie l'image du monde à sa manière et possède une approche conceptuelle unique. Chaque peuple, chaque nation a sa propre vision réelle du monde qui l'entoure, de l'humanité, des gens, des représentants d'autres cultures. Dans toute société, certains stéréotypes se forment sur une personne elle-même, sur le comportement et les traditions dans son espace linguistique et culturel, ainsi que sur d'autres représentants de l'espace linguistique et culturel. Récemment, le phénomène et la notion de stéréotype ont suscité un intérêt croissant chez les scientifiques (Pavlovskaya, 1998; Bergelson, Nekrasova, 2010; Borisova et al., 2015; Egorova et al., 2016).

Afin de définir l'essence de cette notion, les chercheurs s'interrogent sur la légitimité et la justification de l'usage du mot (ou terme) « stéréotype » lui-même. Le stéréotype cognitif est une forme de codage du contenu et du stockage des informations (Krasnykh, 2002), un certain fragment permanent d'une image du monde qui est stocké dans la conscience (Vepreva, 2002). L'un des éléments formant l'image linguistique de ce monde est un stéréotype (Pavlovskaya, 1998).

Récemment, les particularités de la représentation linguistique des stéréotypes dans les médias modernes font l'objet d'une attention particulière et d'un intérêt accru de la part des chercheurs. Et, selon eux, le sens émotionnel et évaluatif du stéréotype devient dominant, ce qui se réalisant, tout d'abord, au niveau du vocabulaire (Pavlovskaya, 1998; Bergelson, Nekrasova, 2010; Borisova, Egorova, 2015 ; Egorova, Kochelaeva, 2016).

V.V. Krasnykh (2002) distingue deux genres de stéréotypes cognitifs : les stéréotypes de comportement et les stéréotypes-perceptions. Selon l'auteur, un type aussi important que les stéréotypes-raisonnement peut être distingué des stéréotypesreprésentations. Cela signifie, tout d'abord, qu'il s'agit d'une interprétation schématique, typique, marquée nationalement, du contenu des concepts culturels (Krasnykh, 2002).

Les stéréotypes cognitifs se trouvent au centre de l'intersection des concepts de base $\mathrm{du}$ discours. Ils représentent une sorte de modèles conceptuels. Les stéréotypes cognitifs sont des unités de pensée, qui définissent les caractéristiques stéréotypées contemporaines et l'évaluation de tel ou tel fragment de la réalité ; ils fournissent toutes les informations sur le phénomène cognitif étudié, qui consiste de valeurs de la structure conceptuelle. 
Le concept est l'unité de base de la linguistique cognitive, il vous permet de corréler le plan mental et le plan verbal dans n'importe quelle sphère linguistique. L'image linguistique de l'espace médiatique est formée par des concepts politiques, économiques et sociaux. Cet article propose l'étude du discours médiatique en utilisant une approche cognitive, dans laquelle le « concept » est la notion clé. Le discours médiatique est étudié dans le cadre de travaux sur le discours et l'analyse critique du discours (Charaudeau, 1983; Fairclough, 2003; Leeuwen, 2008).

À la fin du siècle dernier, il devient clair que le rôle social des femmes dans la société a radicalement changé. Depuis, les recherches sur le rôle des femmes dans le monde des affaires se multiplient (Heilman, Martell, 1986; Helgesen, 1990). En effet, ces dernières années, il y a eu des changements importants dans les concepts liés aux rôles des femmes et des hommes dans la société. En conséquence, il y a des changements dans les concepts connexes tels que " genre » et « leader ». Ces phénomènes peuvent être explorés en se référant aux connotations des représentations verbales de mots tels que " homme », " femme » et " femme d'affaires ». En même temps, les similitudes et les différences entre ces concepts sont étudiées. L'étude de connotation sémantique des relations «femmes et leadership » a été réalisée il y a presque de quinze ans par $\mathrm{S}$. Koch, R. Luff and L. Kruse (2005). Les connotations sémantiques des concepts " femme ", " homme », " leadership », « manager » et " femme d'affaires » ont été étudiées à l'aide de la méthode différentielle sémantique. Les auteurs ont conclu que les rôles sociaux des sexes évoluent dans le sens d'une égalité des sexes.

\section{Méthodologie}

Nous avons étudié 4257 segments discursifs contenant des unités lexicales représentant le concept de "femme d'affaires ». Nombre total de textes médiatiques est de 500. Le matériel a été collecté à partir de publications médiatiques : Le Monde, Le Figaro, Madame Figaro, Marie Claire, Avantages, Vogue, Cosmopolitan et dans d'autres publications.

Afin de mener les recherches, ce matériel a été collecté par la méthode de prévèlement ; la méthode conceptuelle, les principales significations du concept de la valeur culturelle ont été définies et sa structure a été développée à l'aide des caractéristiques les plus fondamentales. Les méthodes de modélisation du cadre ont été utilisées pour identifier les stéréotypes descriptifs-analytiques ainsi que les stéréotypes cognitifs dans la structure du concept, permettant d'observer, d'analyser, de classer les phénomènes linguistiques et d'interpréter les résultats obtenus. Et à l'aide de l'analyse sociolinguistique, les principales données extra-linguistiques de l'émergence et de la formation du concept de femme d'affaires ont été révélées.

La base méthodologique et théorique de l'étude s'est fondée sur les concepts de recherche scientifique des savants et chercheurs suivants :

- en linguistique cognitive : N.D. Arutyunova (1999), V.Z. Demyankov (2001), Yu.D. Apresyan (2006), Z.D. Popova and I.A. Sternin (2010); V.A. Maslova (2011);

- en linguoconceptologie : V.I. Karasik (2004); V.V. Krasnykh (2002); I.V. Privalova (2004); M.V. Pimenova (2011);

- en théorie du discours et en discours des médias : A.N. Vasilyeva (1982); I.P. Lysakova (1989), M.R. Zheltukhina (2003), T.G. Dobrosklonskaya (2005); E.A. Kozhemyakin (2010), G.Ya. Solganik, N.I. Klushina and N.V. Smirnova (2014).

Dans la littérature scientifique moderne, différents termes sont utilisés en relation avec le concept de stéréotype : stéréotypes nationaux, stéréotypes culturels, préjugés ethniques, attitudes ethniques, images nationales, etc. (Ufimtseva, 1995; Elizarova, 2005; Privalova et al., 2019; Tameryan et al., 2019). La plupart des études menées se sont concentrées sur les stéréotypes ethniques, c'est-à-dire que de simples images des

XLinguae, Volume 14 Issue 4, October 2021, ISSN 1337-8384, eISSN 2453-711X 
communautés ethniques (ethnies) sont considérées de manière globale. Cependant, la diversité des communautés sociales actuelles pose constamment le problème de la définition de frontières claires entre la sienne propre et celle d'autrui. Ainsi, le terme de stéréotype est largement utilisé pour analyser les particularités de la perception humaine entre une personne particulière et une autre qui diffère selon ses opinions politiques, ses croyances religieuses, sa culture, sa profession, son âge, sa génération, son genre, ou encore son statut économique et matériel.

Le stéréotype national, inhérent à un certain groupe national ou à la nation dans son ensemble, détermine les traits relatifs à tous les représentants de cette nation, et acquiert un certain caractère évaluatif, implicite ou explicite. Les stéréotypes nationaux sont étudiés par de nombreuses branches, comme la sociologie, la psychologie, les sciences politiques, la philologie, l'histoire, etc.

Du point de vue de la linguistique cognitive et des études linguistique culturelle, le terme stéréotype est interprété comme une catégorie mentale, de pensée, identique à l'image du monde et reflétant l'essence sémantique de la langue et de la culture. «Le monde réel existe objectivement. Ce monde est affiché dans la psyché humaine de telle manière que cet affichage est une image du monde ou une image conceptuelle du monde. La verbalisation de l'image conceptuelle du monde est une image linguistique du monde » (Privalova, $2004: 54$ ). L'image du monde, y compris l'image linguistique du monde, est basée sur les idées de l'individu sur l'univers, sur l'environnement. Les résultats de la transformation des informations sur une personne et son environnement se reflètent dans une image du monde. Une image du monde est une compréhension particulière du monde, le résultat d'une remise en question produite par l'homme. L'image du monde n'est pas statique, elle est toujours sujette à des changements. Par conséquent, selon les chercheurs en linguistique cognitive, notre système conceptuel, qui est une image linguistique du monde, est directement lié à notre expérience physique et culturelle, à notre mode de vie et mentalité.

En général, les causes d'apparition des stéréotypes sont considérées individuellement dans les théories cognitives et dans la psychologie sociale. La question principale de ces domaines est de savoir dans quelle mesure notre raisonnement est influencé par le contexte social ? Comment cela affecte-t-il notre comportement dans la société ?

L'image linguistique du monde et le stéréotype linguistique sont identifiés comme un ensemble, et comme une partie de celui-ci. À cet égard, un stéréotype linguistique est défini comme un jugement. Il présente des caractéristiques et des éléments d'évaluation. Les stéréotypes linguistiques comprennent non seulement les jugements, mais aussi tout énoncé stable (cliché, phrase figée, comparaison, etc.).

$\mathrm{Au}$ cours de l'analyse linguistique cognitive des principaux concepts de tout discours en langue nationale, l'utilisation de stéréotypes comme unités opérationnelles de base est considérée comme tout à fait justifiée : premièrement, elle permet de révéler les composantes nationales de l'interprétation du contenu stéréotypé dans les textes médiatiques ; deuxièmement, elle permet de définir les spécificités de la représentation linguistique dans le discours.

Des catégories telles que la structure cognitive, le concept, le modèle conceptuel et le stéréotype cognitif se reflètent dans la langue et le discours. Ils agissent comme des marqueurs lexicaux et lexico-syntaxiques des stéréotypes cognitifs.

\section{Résultats}

Le concept de femme d'affaires en tant que structure mentale complexe reflète un grand nombre de caractéristique cognitives. Le processus de formation de l'image de la femme d'affaires dans les textes médiatiques français, s'effectue simultanément avec le développement de stéréotypes à son sujet. Des vues standardisées des femmes d'affaires apparaissent dans la conscience de la société. Le modèle cognitif du concept de femme d'affaires peut être représenté comme une structure cadre et se compose de 
fentes définissant une dépendance hiérarchique : activité professionnelle, apparence, caractéristique morale, compétences et aptitudes commerciales, valeurs sociales, matérielles et spirituelles, etc.

Les représentations typiques de la réalité sont déterminées par des stéréotypes cognitifs. Ils constituent la structure sémantique du concept de femme d'affaires dans le discours médiatique français. Les particularités des représentations des Français sur la femme d'affaires sont conditionnées par des traditions culturelles et historiques. En général, le processus de formation du concept de femme d'affaires dans le discours médiatique s'effectue selon les analogies binaires suivantes : féminité, tendresse (qualités féminines) / masculinité, virilité (qualités masculines). L'image de la femme d'affaires moderne dans le discours médiatique est créée par des moyens linguistiques, qui expriment certaines qualités, compétences et capacités. Leur choix est déterminé par la possibilité d'influencer efficacement la conscience de masse.

Les raisons de la formation de l'image de la femme d'affaires en français sont : premièrement, une motivation intrinsèque, c'est-à-dire la manifestation d'un intérêt pour tel ou tel type d'activité ; deuxièmement, la possibilité de créer sa propre entreprise (dans ce cas, la principale profession des femmes est, par exemple, comptable - travail, activité, profession, et entrepreneuriat, passe-temps, loisirs).

\section{Discussion}

\section{Les facteurs extra-linguistiques de formation du concept de femme d'affaires}

\subsection{L'émergence du concept de femme d'affaires}

En 1988, après le film Working girl de Michael Nicholas, le concept de femme d'affaires a émergé dans la conscience de la langue russe (Kokova, 2017 : 107). En français, le terme féminisme a été inventé en 1837 par le socialiste et savant français Charles Fourier, qui a également introduit le terme dans la culture politique.

À cet égard, après l'émergence de la désignation linguistique de ce concept, des mouvements féministes se sont activement développés en France et en GrandeBretagne. Et après un certain temps, en 1866, le mouvement européen mentionné commence à apparaître aux États-Unis. Dans ce pays, ce mouvement s'est développé très lentement, car à la fin du vingtième siècle, la communauté des femmes souffrait de discrimination. En France, en revanche, l'émancipation des femmes a été pleinement réalisée dans les années 20-30 du vingtième siècle (Gavrilov , Ryabtseva, 2000).

Par conséquent, la tâche principale est d'identifier tous les traits sémantiques des unités linguistiques de femme d'affaires car ces noms font référence à nombre de concepts qui ne sont inclus dans aucun dictionnaire. Par conséquent, leurs définitions n'ont pas été définies ou développées. La première fixation du lexème businessman en anglais a été effectuée en 1840. Le sens original d'un ancien mot anglais bisignisse (dialecte anglais de Northumberland) signifiait emploi, affaires, danger (soin, anxiété) (Dictionary.com, 2020). En définitive, à la fin du vingtième et au début du vingt-etunième siècle, le contenu du mot "homme/femme d'affaires" se compose des significations suivantes :

1) une personne d'affaires ; le/la créateur(-trice) et le/la fondateur(-trice) d'une entreprise ;

2) un entrepreneur (une entrepreneuse) exerçant tout type d'activité économique légitime qui génère des revenus ;

3) un(e) homme/femme d'affaires ; un entrepreneur (une entrepreneuse) ; engagé(e) dans une activité entrepreneuriale privée, un commerce ; une personne qui a sa propre entreprise.

\subsection{Utilisations significatives du concept de femme d'affaires}

XLinguae, Volume 14 Issue 4, October 2021, ISSN 1337-8384, eISSN 2453-711X 
Les noms femme d'affaires (businesswoman / businesswomen) au sens de femme d'affaires sont des corrélats de genre du lexème de businessman : businesswoman, businesswoman (en anglais). Par conséquent, les mots et combinaisons de mots homme d'affaires et femme d'affaires ou entrepreneur(entrepreneuse) et femme d'affaires, femme ayant sa propre entreprise ne diffèrent les uns des autres que par un marqueur de genre. Après tout, une femme d'affaires est une femme engagée dans le commerce, l'entrepreneuriat.

Sur cet ascpect, un des points très importants de notre travail réside dans l'incapacité de distinguer le concept de notre étude d'une autre image féminine, qui se forme à travers les textes médiatiques. À son tour, elle est également considérée comme un concept - une femme qui réussit. Les concepts susmentionnés se différencient tout d'abord par le lieu de publication des textes médiatiques, alors que la grande majorité des textes médiatiques concernant le concept de femme d'affaires apparaissent dans les pages des journaux. Les textes médiatiques représentant le concept de femme à succès sont publiés dans des magazines spéciaux sur papier glacé ciblant principalement le public féminin. Ils se caractérisent par une forte demande et une forte concurrence, des couleurs vives, des images, une grande attention accordée à l'esthétique, etc. Ils constituent le type de texte médiatique le plus populaire. Le concept de la femme qui réussit est formé par l'image créée dans l'esprit des gens modernes par les textes médiatiques.

Les principales caractéristiques des textes médiatiques visent à créer une image généralisée d'une femme qui a réussi : belle, populaire, qui fréquente les hautes sphères, riche, etc. et le lecteur ne se soucie pas vraiment de savoir de quelle manière, de quelle façon elle a obtenu un tel succès.

La langue de ces textes médiatiques est interconnectée avec les normes qui reflètent les traditions de la culture linguistique d'une nation. Leur objectif est de créer une certaine expression. La langue des textes médiatiques se réalise par l'affranchissement des normes d'expression.

L'un des résultats de l'interaction entre la culture et les médias est la popularisation de la culture. Pour cette raison, la plupart des experts estiment que le langage de la communication de masse est de plus en plus simplifié et ne correspond pas aux normes linguistiques. Les journalistes russes observent ce phénomène avec inquiétude, notant que la langue des textes médiatiques s'est éloignée de son caractère national pour devenir un moyen de communication global (Vepreva, 2002; Klushina, 2012; Solganik, Klushina, Smirnova, 2014).

À notre avis, les sujets consacrés à la mode, aux cosmétiques, aux bijoux, au fitness et à la santé, etc. sont principalement axés sur les femmes qui réussissent dans la société. Il est légitime de penser que la norme mentionnée dans le discours médiatique moderne a un impact énorme sur les femmes modernes, modifiant leur comportement, leurs stéréotypes de pensée, leur rôle dans la société. Il est connu que même les nouveaux points de vue sur la vie se forment dans la culture linguistique. Par exemple, dans l'article suivant il existe le sens de la propagande qu'on peut avoir la réussite facile, et devenir riche sans aucune difficulté :

Selfies, gloire et beauté : les stars millionnaires de la cosmétique. En avril 2015, elle poste un selfie, la bouche gonflée à bloc, niant tout recours à la médecine esthétique. Les ados qui suivent à la lettre ses conseils lancent le «Kylie Jenner lip challenge " et, avec des verres à shot, provoquent un effet ventouse qui pulpe les lèvres. Au final, si la starlette admettra avoir eu recours à des injections, elle profitera de ce buzz pour lancer des kits de maquillage pour la bouche, qui seront épuisés en une minute. Quatre ans plus tard, Kylie Jenner a 132 millions d'abonnés sur Instagram. Sa marque, Kylie Cosmetics, s'est bien développée. Elle propose en ligne toute une gamme de produits, pour les lèvres, le teint ou les yeux. En juillet 
2018, elle faisait même, à 21 ans, la couverture du magazine économique Forbes, qui la sacrait, en se fondant sur la valeur estimée de son entreprise, "plus jeune selfmade-milliardaire de tous les temps", dépassant le record établi à son époque par Mark Zuckerberg à l'âge de 23 ans avec Facebook. En signant cette année avec les magasins américains de cosmétiques Ulta Beauty, Kylie Jenner, qui détiendrait, selon Bloomberg, $90 \%$ de sa marque (les $10 \%$ restant étant aux mains de sa mère), devrait encore engranger de nombreux profits (Dhouailly, 2019).

Les principales différences entre les images de la femme d'affaires et de la femme à succès dans les textes médiatiques français sont les suivantes : premièrement, les caractéristiques sémantiques, qui reflètent le contenu du concept de femme d'affaires :

- Elle a une grande capacité d'organisation, elle a le sens des affaires, elle est très instruite, elle aime son travail, etc. // une femme qui a du succès est célèbre, aisée, riche, a du succès dans sa carrière, est habillée à la mode, vit dans une bonne maison, un bon appartement, etc.

- Deuxièmement, lorsque nous parlons d'une femme d'affaires, nous parlons de la manière dont elle a atteint le succès (échecs de la vie, difficultés, etc.) // la manière dont une femme qui a du succès est devenue plus performante n'est pas mentionnée. Il ne s'agit pas de coûts spirituels, physiques, matériels, et son succès n'est donné que comme un résultat.

- Troisièmement, la représentation du concept de femme d'affaires dans le texte médiatique coïncide entièrement avec la signification du concept - réussie, active, confiante, ayant un revenu stable, une connexion avec diverses institutions sociales.

L'image d'une femme qui réussit est principalement définie par la signification : efficacité, efficience de toute activité, mais pas nécessairement l'expérience des échecs, des difficultés sur la voie du succès, c'est-à-dire que les composants sémantiques du concept sont utilisés.

2 La formation du concept de la femme d'affaires dans la culture linguistique française

\subsection{Quelques caractéristiques comportementales des Français}

Récemment, la littérature scientifique linguistique a affirmé que chaque nation a son propre caractère quand il s'agit du comportement. Par exemple, les Américains sont efficaces, les Kazakhs hospitaliers, les Allemands ont une ponctualité exceptionnelle, les Français un langage tranchant et de la politesse, de même que l'édification à une grande importance pour eux, etc. Cependant, les avis des scientifiques sont partagés : certains pensent que le comportement national est une vérité reflétée dans l'histoire, le mode de vie et la culture, la psychologie de la nation ; selon d'autres, le trait de personnalité national est perçu comme un stéréotype psychologique figé. Un autre groupe d'universitaires considère le comportement national comme une véritable réalité (Cart , Fox, 2005 : 17).

Le comportement des Français varie en fonction de leur caractère, statut social. Mais il est possible de distinguer plusieurs caractéristiques communes inhérentes à l'ensemble de la nation : patriotisme, esprit d'initiative, rationalité, tendance à exprimer sa pensée de manière claire, logique et précise, capacité à défendre son point de vue, manière gracieuse et artistique de s'exprimer, etc. En général, la France est considérée comme l'un des États qui ont eu une grande influence sur la formation européenne de modèles de comportement. Un très haut niveau d'éducation, qui a trouvé une combinaison parfaite de capacités analytiques et de pragmatisme naturel, est devenu la base de la formation d'entreprises performantes et rentables des Français.

XLinguae, Volume 14 Issue 4, October 2021, ISSN 1337-8384, eISSN 2453-711X 
Les fonctions historiques et traditionnelles des femmes dans la société française comprennent l'entretien du foyer, la garde des enfants, la cuisine, etc. Ce n'est qu'après la révolution industrielle en France que le rôle des femmes subit plusieurs changements : les femmes commencent à apparaître parmi les propriétaires d'entreprises. L'émancipation des femmes françaises en 1944 a renforcé leur place et leur rôle dans la société. Et depuis les années 1960, elles ont le droit de trouver un emploi et d'ouvrir un compte bancaire personnel sans l'autorisation de leur mari. Dans la France d'aujourd'hui, les femmes qui ont atteint le niveau d'éducation nécessaire occupent des postes de premier plan, tant dans dans différents sectuers économiques.

Ainsi, nous pouvons conclure que dans la culture linguistique française, le concept de femme d'affaires commence à se former au milieu du $\mathrm{XX}^{\text {ème }}$ siècle. En effet, c'est alors dans la sphère économique de l'État que les premières femmes-entrepreneuses ont commencé à travailler dans diverses sphères de l'industrie : production de cosmétiques, construction mécanique, etc.

\subsection{Les caractéristiques des femmes d'affaires dans la culture linguistique française}

Les femmes d'affaires sont classées en deux catégories : les gestionnaires et les femmes-entrepreneuses. Par conséquent, elles sont déjà divisées en deux autres groupes : les managers qui font leur propre carrière et les managers professionnels. Quant à la définition de la femme d'affaires en français nous trouvons : femme d'affaires (n.f.) - celui qui fait profession de gérer des entreprises ou d'en détenir le contrôle (Le Parisien, 1998).

Les synonymes de ce concept en français sont : homme/femme d'affaires, dirigeant(e), personne ayant une responsabilité dans une entreprise, chef d'entreprise. Parmi les catégories de femmes énumérées, il existe des différences dans la réalisation de l'objectif de carrière fixé. Dans ce cas, le concept de femme d'affaires est entendu comme une femme exerçant une activité commerciale sous la forme d'une entreprise privée et également responsable d'un poste au sein d'une certaine entité juridique ; ayant le droit de prendre des décisions sur la politique stratégique de l'entreprise qu'elle dirige. En même temps, la structure de la notion de femme d'affaires dans la culture linguistique française inclut un groupe de femmes dans la fonction publique. Chacune d'entre elles a des tâches de gestion différentes, des particularités de l'environnement externe et des caractéristiques individuelles mutuellement exclusives propres à ce groupe. À cet égard, les extraits d'articles suivants peuvent servir d'exemples de ce qui précède.

Et Christine Nagel a pour mission de l'incarner. Le visage de cette femme élégante de 59 ans, au regard gourmand malgré d'épaisses lunettes, était déjà connu des magazines avant sa nomination au sein d'Hermès. Me Nagel n'a pourtant pas le pedigree des nez qui travaillent pour les grandes maisons. Elle n'est pas née à proximité des alambics. Chez Chanel, Olivier Polge a succédé à son père en 2015, en tant que nez exclusif de la maison connue pour son $N^{o}$ 5. François Demachy, parfumeur-créateur chez Christian Dior, a, quant à lui, été élevé à Grasse (AlpesMaritimes). Jacques Cavallier-Belletrud, maître parfumeur chez Louis Vuitton depuis 2012, fils et petit-fils de parfumeurs, est né dans ce berceau de la parfumerie hexagonale. Enfin, chez Guerlain, Jean-Paul Guerlain a transmis le livre des formules de la maison mère de Shalimar à Thierry Wasser. En 2008, il en a fait son fils spirituel. Rien de tel pour Christine Nagel : elle n'est pas " fille de », n'a pas suivi de formation en parfumerie et n'est pas originaire de Grasse. De surcroît, elle est femme dans un microcosme dominé par les hommes - on ne compte que 500 nez dans le monde, c'est moins que le nombre d'astronautes, rappelle-t-elle (Garnier, 2019). 
...les femmes travaillent davantage. Pour moi, c'est une des choses essentielles : la femme doit avoir son indépendance économique... Seulement cela n'empêche pas qu'elle concilie toujours son travail à la maison...Il faut que ce soit elle, même si elle est un grand patron, une chirurgienne réputée, il faut que ce soit elle qui fasse ensuite le ménage, la cuisine, mette le couvert, etc., qui fasse les courses, et elle a donc une double charge (Viansson- Ponté, 2019a).

Simplement, il arrive très souvent que, si les femmes réussissent sur ce plan-là, elles se désolidarisent des autres femmes et elles pensent que, si elles ont réussi, n'importe qui peut réussir. Or c'est complètement faux parce que, si l'on réussit, c'est par des chances, ou de naissance, ou de vie, ou d'argent, et cela ne prouve pas du tout que la situation des femmes en est changée (Viansson-Ponté, 2019).

En d'autres termes, pour une femme d'affaires dans la culture linguistique française n'a pas l'obligation d'être mariée ou d'avoir une famille ou des enfants : tout cela est relégué au second plan ou non, et on oublie même souvent que la personne responsable d'une entreprise est une femme, on ne fait pas d'attention au sexe de la personne responsable d'une entreprise. Cela signifie que les femmes d'affaires sont tout à fait prêtes à affronter la concurrence, à se battre, et en cas d'échec, elles ne se découragent pas, mais relèvent de nouveaux défis :

Danièle Kapel-Marcovici, femme d'affaire et de combats. D'une petite entreprise d'emballage carton fondée par sa mère, Danièle Kapel-Marcovici a fait une des grandes forces industrielles françaises. Et elle reverse chaque année une partie $d u$ bénéfice à des femmes qui en aident d'autres (Edgard-Rosa, 2020).

Les femmes d'affaires ont une très haute opinion d'elles-mêmes. Elles sont dominantes en comptant autant que possible sur leurs capacités. Une femme d'affaires est très intelligente, soignée, totalement indépendante et autosuffisante sur le plan financier. Les femmes d'affaires françaises sont souvent les pionnières de mode et les arbitre des élégances :

La marque de prêt-à-porter et l'influenceuse Camille Callen se sont associées pour concocter des looks de femmes d'affaires chic et mode (Sèbe, 2020).

En tant que personne, elle a beaucoup de pensées créatives, elle est socialement importante et elle s'intéresse beaucoup à elle-même ; son mode de vie est très actif, elle est capable de prendre des décisions rapides, elle cherche à atteindre de grands sommets dans sa carrière, elle est capable de se suffire à elle-même et de montrer qu'elle est au-dessus des autres (Novikova, 2009: 4). Il est fréquent de soutenir qu'elle est une telle personne, capable de combiner les valeurs et les qualités inhérentes aux hommes et aux femmes : sens des affaires, érudition, inclination à prendre une direction différente dans la prise de décision sur des questions complexes d'ordre économique, politique, etc., disposition à intervenir dans un nouveau domaine d'activité, qualité d'organisation et d'exécution bien soignées, capacité à prendre des décisions efficaces dans des situations difficiles (Isachenko, $2005: 5$ ).

Selon A.M. Kurkimbayeva et autres (2020) la mise en œuvre de la stratégie de référence aux conseils dans les blogs de voyage peut également être un facteur clé dans l'exploration du concept de femme d'affaires dans toute culture linguistique, car des milliers de destinataires se tournent vers leurs conseils, comment réussir dans le monde des affaires (Kurkimbayeva et al., 2020).

\section{Le concept de femme d'affaires dans le discours médiatique français}

\subsection{Les principales caractéristiques de la catégorie de concept}

L'opinion généralement admise par la majorité des scientifiques est que le concept comporte les éléments suivants :

XLinguae, Volume 14 Issue 4, October 2021, ISSN 1337-8384, eISSN 2453-711X 
- la composante universelle du concept - les caractéristiques sémantiques qui sont communes au concept dans de nombreuses langues ;

- la composante nationale d'un concept - les caractéristiques sémantiques, liées à la mentalité de la personnalité linguistique nationale ou à la mentalité des locuteurs natifs et communes même pour une partie de la représentation linguistique d'un concept avec des caractéristiques de la culture linguistique, ethno-sémantiques ;

- composante personnelle d'un concept - caractéristiques cognitives, définies par l'individu, sa propre expérience (ces composantes structurelles sont particulièrement importantes pour l'étude des concepts artistiques).

Mais tous les concepts n'ont pas le même contenu que celui mentionné ci-dessus. L'un de ces concepts, qui comprend les trois composantes identiques du concept susmentionné, est le concept de femmes. C'est l'un des concepts de base de l'image du monde. Après tout, la femme-mère est considérée comme la caractéristique principale de ce concept de caractère universel. C'est la femme-mère qui, pour la première fois, forme dans la conscience de son enfant les fondements de l'image de l'univers inhérente à sa nation et à son peuple. La maternité est la fonction la plus importante des femmes dans le monde.

Le sens universel du concept français de femme se compose des éléments sémantiques suivants :

- beauté : La plus belle femme ne peut donner que ce qu'elle a (Le Monde. fr) ;

- maternité : "L'instinct maternel est souvent perçu comme une sorte de "superpouvoir" que possèdent les mamans et qui leur permet souvent de mieux comprendre leur nourrisson» (Capronnier, 2019);

- sagesse : La femme fait la maison ; C'est la bonne femme qui fait le bon mari, Femme bonne vaut couronne (Journal Tania-Soleil).

Les caractéristiques spécifiques comprennent : La femme est comme la châtaigne, belle au-dehors et dedans le ver, La femme est la porte de l'enfer (Tertullien) (Le Figaro.fr) (transmission simultanée de qualités spirituelles et extérieures : femme dispensatrice des forces du mal, pasteur, prédicateur ; seigneur du mal ; clé des portes de l'enfer, etc.). En même temps, les caractéristiques universelles constituent le noyau de la structure du concept, tandis que les caractéristiques spécifiques appartiennent aux unités périphériques.

Les caractéristiques conceptuelles sont obligatoires dans la structure d'un concept. Cela signifie qu'afin de dériver un modèle de n'importe quel concept, il est nécessaire, tout d'abord, de déterminer quels sèmes de base composent sa couche conceptuelle. Ces sèmes constituent le cœur du concept. Et sur la base de l'analyse sémantiquecadre, il est possible de définir la conformité d'un concept avec d'autres types de cadres, tels que le sujet, l'objet, les relations causales, les buts, la manifestation de l'activité, et aussi de révéler les signes périphériques d'un concept objectivé dans une variété de discours.

3.2 Les caractéristiques de base des concepts de discours, discours médiatique, texte médiatique

Il est indiscutable que les études consacrées aux problèmes de l'appartenance sociale et de la concrétude du discours dans le contexte de l'orientation discursive ont reçu une suite dans la considération de l'expérience de la parole et ont commencé à se développer au sein de la méthode linguistique moderne visant l'étude de la communication de la parole. Conformément à cela, on peut noter que dans la littérature scientifique moderne, le terme de discours est très souvent utilisé, dans le sens par le terme d'expérience discursive. 
Par conséquent, en particulier, le discours est un système de contraintes sociales et idéologiques. Nous parlons de types de discours spécifiques, de leurs nombreuses manifestations : discours religieux, discours politique, discours médical, discours économique, discours publicitaire, discours pédagogique, discours médiatique (massmedia), etc. Aujourd'hui, il est évident que ce sont ces critères et limites du discours qui sont au cœur de la recherche.

L'objet de notre étude, orienté vers la problématique de la communication de masse, nécessite de prendre en compte les propriétés médiatiques spécifiques du discours. Le discours médiatique est un phénomène complexe. Il se définit, tout d'abord, par son étroite continuité avec les médias et la communication.

En général, le contexte communicatif reflète les caractéristiques du discours médiatique dans un état statique, et définit les composantes socioculturelles des événements dans le texte médiatique par les médias.

Sans aucun doute, le problème de l'interconnexion des concepts de texte médiatique et de discours médiatique est d'actualité aujourd'hui. À son tour, la communication médiatique se caractérise et possède de grandes capacités techniques de journalisme. Celle présente les mêmes informations à l'oral et à l'écrit en même temps.

Les particularités du discours oral et écrit, entraînent des difficultés par rapport à certains genres médiatiques, par exemple : rapport analytique, entretien polémique, interview, etc. Comme nous le savons tous, ces genres médiatiques sont enregistrés sur vidéo en tant que faits de discours oraux et diffusés sous forme écrite au grand public. Le problème est également lié à la définition du texte médiatique et du discours médiatique. Par conséquent, les chercheurs en langue des médias considèrent que le texte médiatique et le discours médiatique sont étroitement liés au contexte de communication, et aux connaissances de base de l'énonciateur, de la personne qui produit le discours (Zheltukhina et al., 2017).

Les textes médiatiques doivent être considérés comme des unités du discours médiatique, qui sont à la fois le résultat et l'outil de l'expérience discursive des médias. Le médiatexte est une catégorie de base de la linguistique des médias. Cette catégorie influence directement la sélection, qui a la fonction d'organisation des éléments principaux sur les niveaux interconnectés de la structure et du contenu linguistiques. Par exemple, la scientifique M.R. Zheltukhina (2003) classe le langage des médias selon les systèmes de leur influence :

1) les fonctions cognitives, culturelles, créatives sont distinguées dans le système expéditeur -destinataire ;

2) les fonctions représentatives, expressives et réglementaires sont distinguées dans le système d'expéditeur ;

3) les fonctions hédonistes, harmonisantes et intégratives sont distinguées dans le système du destinataire (Zheltukhina, 2003).

Selon M. Zheltukhina et autres (2017) : La culture des médias de masse met en œuvre l'impact sur le destinataire en raison d'une combinaison des trois mondes : réel, informationnel (mémoire individuelle) et symbolique (mémoire sociale). La signification de tout événement linguistique-culturel est notée dans les médias. Les sources d'information sont les systèmes éducatifs (connaissances, mythes), les religions (mythes, foi), la propagande, la publicité, la culture, principalement la culture de masse (mythes, messages) (Zheltukhina et al., 2017).

La particularité du destinataire du discours médiatique est son appartenance directe à la situation pragmatique, et elle nécessite à son tour un impact sur l'acte de parole. Tout message est transmis par l'expéditeur par le biais de divers canaux de communication et d'information et uniquement à une partie clairement orientée de la société.

XLinguae, Volume 14 Issue 4, October 2021, ISSN 1337-8384, eISSN 2453-711X 
En raison de ce processus continu, de nouvelles tendances et approches de l'étude du langage apparaissent dans la science contemporaine. Après tout, au sens large, l'espace médiatique est l'environnement dans lequel se déroule le discours médiatique.

\subsection{Les marqueurs linguistiques du stéréotype cognitif de la femme d'affaires}

À l'heure actuelle, le développement du concept de description du cadre est particulièrement pertinent. Une situation stéréotypée est représentée par des cases de cadre. Les cadres sont définis par la mesure dans laquelle ils coïncident avec les concepts du système conceptuel d'une personne. Toutes les unités réalisées au sein d'un certain concept sont appelées cadres. Les unités de cadre reflètent les informations de base typiques et possibles qui sont associées à un certain concept. Un cadre est la base d'une situation ou d'un concept stéréotypé. Il représente les significations cognitives dénotées par le langage - images, vues, concepts, etc. La structure du cadre des unités cognitives est une condition nécessaire à leur matérialisation verbale. Elle favorise l'interaction des sphères mentales et matérielles. Par conséquent, un cadre est défini comme une structure hiérarchique et comporte plusieurs niveaux interdépendants.

L'analyse de la signification d'une unité lexicale sous l'aspect cognitif implique de déterminer le domaine de connaissance.

Il est important de construire un cadre qui définit cette signification. L'analyse du cadre contribue à l'identification des caractéristiques conceptuelles spécifiques d'un concept. L'analyse conceptuelle reflète les particularités de la conceptualisation de la réalité environnante dans la sémantique des unités linguistiques.

En ce qui concerne les spécificités de fente française de la sphère des affaires, l'entreprise continue d'être héritée du père : L'histoire d'Alsapan, est celle d'une aventure entrepreneuriale singulière. Fondée en 1972, par Joseph Strub, menuisierébéniste, développée ensuite par ses trois enfants, elle est aujourd'hui dirigée par sa petite fille, Cécile Canterelle... Alsapan possède aujourd'hui cinq sites de production et emploie 800 salariés. Cécile Canterelle, la dynamique dirigeante d'Alsapan, travaille étroitement avec la Banque Kolb, qui a accompagné la croissance de l'entreprise (Cantrelle, 2019).

Dans le texte médiatique, une femme d'affaires est une femme qui possède sa propre entreprise, qui est créé par elle-même ou qui représente une entreprise familiale qu'elle a créée ou héritée. La structure de base du contenu des fentes "sphère de gestion" est la suivante : directeur de banque, président d'entreprise, président de société, ministre, rédacteur en chef de magazine, etc. Par exemple : La nouvelle a fait sensation. Apple a annoncé, mardi 5 février au soir, le départ de la responsable des Apple Stores Angela Ahrendts. Elle était l'une des figures les plus en vue de la firme de Cupertino. Et de l'avis de certains, peut-être la mieux placée des dirigeants d'Apple pour succéder à Tim Cook, à la tête de la société depuis plus de vingt ans (Fagot, 2019); Catherine Guillouard, PDG de la RATP depuis 2017, était l'invitée, jeudi, du Club de l'économie du "Monde ». Elle s'explique sur les défis historiques que doit relever l'entreprise publique (Béziat, Escande, 2019); Le train rapide entre Paris et l'aéroport de Roissy sera Bel et bien construit, malgré l'inquiétude des usagers et l'opposition des principaux élus d'Ile-de-France. La ministre des transports, Elisabeth Borne, a confirmé, mardi 5 février, dans un entretien au Parisien, que le CDG Express serait mis en service pour les Jeux olympiques de 2024 (Allix, 2019).

Il convient de noter qu'il existe des exemples dans le discours des médias français selon lesquels les femmes d'affaires sont plus susceptibles de rencontrer des difficultés que les hommes d'affaires. Il existe encore des stéréotypes et des préjugés sur l'égalité des chances entre les hommes et les femmes: 
Les conseils de quatre femmes d'affaires pour relever haut la main le défi de la levée de fonds. Si l'argent est le nerf de la guerre, la bataille ne se joue pas à armes égales. Convaincre des investisseurs est un parcours du combattant encore plus ardu quand on est une femme (Chocas et al., 2019).

En résumé, la formation de l'image de la femme d'affaires moderne dans la conscience de masse et sa popularisation se produisent sous l'influence des médias. En général, l'un des concepts linguaculture les plus importants est celui de femme d'affaires. Parce qu'il reflète les perceptions stéréotypées de la femme d'affaires moderne.

\section{Conclusion}

La femme d'affaires dans la culture linguistique française met son âme féminine au second plan (complètement oubliée), privilégiant le personnage masculin au détriment du mariage, de la fondation d'une famille et de l'éducation d'enfants. En d'autres termes, cela signifie que les femmes d'affaires sont tout à fait prêtes à concourir, à se battre et, en cas d'échec, à ne pas se décourager, mais à se battre à nouveau. En effet, les facteurs suivants influencent la formation de ce concept, dans la culture linguistique française : aspirer à atteindre de grands sommets dans sa carrière, prouver sa capacité à se suffire à soi-même, s'élever au-dessus des autres, etc.

L'analyse linguistico-cognitive des principaux concepts discursifs dans la langue a permis d'établir que la principale unité opérationnelle est constituée des stéréotypes de raisonnement, qui sont verbalisés dans les types de discours linguistiques les plus différents. Les marqueurs linguistiques des stéréotypes cognitifs dans le texte médiatique sont les suivants : des énoncés semi-clichés à haute fréquence d'utilisation, correspondant à des structures propositionnelles, divers segments de texte, à savoir une combinaison de mots, plusieurs mots apparentés qui verbalisent la structure du contenu du stéréotype, etc.

Ainsi la femme d'affaires, du point de vue de la culture sociale est reconnue comme une actrice active de la culture, capable de combiner les valeurs inhérentes aux hommes et aux femmes dans sa nature unique. Dans le texte médiatique, la femme d'affaires est présentée comme une femme qui possède sa propre entreprise, qu'elle a créée elle-même ou qui représente une entreprise familiale qu'elle a créée ou héritée.

\section{Acknowledgments}

This paper has been supported by the RUDN University Strategic Academic Leadership Program.

\section{Bibliographic references}

ALLIX, G. 2019. Le CDG Express confirmé malgré les risques pour les usagers du Rer B. In: Le Monde. Available online: https://www.lemonde.fr/economie/article/2019/02/05/le-cdg-express-reliant-paris-a-1aeroport-de-roissy-sera-construit-confirme-la-ministre-destransports_5419675_3234.html ISSN 0395-2037

APRESYAN, Yu.D. 2006. Language picture of the world and system lexicography. Moscow: Languages of Slavic cultures. (Studia philologica). ISSN 1726135X, ISBN 5-9551-0135-7

ARUTYUNOVA, N.D. 1999. Language and the human world. Moscow: Languages of Russian Culture. ISBN 5-7859-0027-0

BERGELSON, M.B. - NEKRASOVA, A.E. 2010. Linguistic analysis of stereotypes: a balance between texts and meanings. In: Collection of articles based on the materials of the conference "Computational Linguistics and Intelligent Technologies", vol. $9, \quad$ n. $16, \quad$ pp. 30-35. Available online: https://www.hse.ru/data/2012/03/18/1264909488/book_dialog2010.pdf 
BEZIAT, E. - ESCANDE, Ph. 2019. Catherine Guillouard: «La RATP investit chaque année un tiers de son chiffre d'affaires ». In: Le Monde. Available online: https://www.lemonde.fr/economie/article/2019/02/01/la-ratp-investit-chaque-anneeun-tiers-de-son-chiffre-d-affaires_5417560_3234.html. ISSN 0395-2037

BORILLO, A. 2006. Quelques structures participiales de valeur temporelle en prédication seconde. In: Travaux Linguistiques du CerLiCO, vol. 19, pp. 167-182. ISBN 2-7535-0268-4

BORISOVA, S.A., - EGOROVA, E.V. - KRASHENINNIKOVA, N.A. OSETROVA, O.I. 2015. Stereotype in the structure of political communication. In: In the world of scientific discoveries, vol. 7, n. 67. pp. 642-653. ISSN 2330-927X

BORISOVA, S.A., - EGOROVA, E.V. 2015. Stereotype about the Slavic brotherhood in the Russian media space. In: In the world of scientific discoveries, vol. 7, n. 67, pp. 2891-2899. ISSN 2330-927X

CANTRELLE, C. 2019. Alsapan. Available online: https://www.lemonde.fr/parolesd-entrepreneurs-au-coeur-des-territoires/article/2019/02/27/cecile-cantrellealsapan_5429048_5428808.html. ISSN 0395-2037

CAPRONNIER, M. 2019. L'instinct maternel : est-il inné et commun à toutes les femmes? In: Journal des Femmes. Available online: https://www.journaldesfemmes.fr/maman/guide-des-parents/2578611-instinctmaternel/ ISSN 2608-9394

CART, P. - FOX, K. 2005. Overcoming cultural differences. A practical guide to business international business communication. Moscow: FLIR-PRESS. ISBN 58183-0991-6

CHARAUDEAU, P. 1983. Langage et discours. Paris: Hachette université. Coll. Langue, linguistique, communication. ISSN 0768-2832.

CULIOLI, A. 1999. Pour une linguistique de l'énonciation. Formalisation et opérations et repérage. Paris: Ophrys. ISBN 9782708009004.

CHOCAS, V. - KHIDER, M. - MIEL, M. - BARON. Y. 2019. Les conseils de quatre femmes d'affaires pour relever haut la main le défi de la levée de fonds. In : Madame Figaro, Le 18 avril 2019. Available online: https://madame.lefigaro.fr/business/leveede-fonds-lenjeu-des-femmes-prix-business-wtih-attitude-entreprendre-start-upinvestissement-160419-164736 ISSN 0182-5852

DEMYANKOV, V.Z. 2001. Notion and concept in fiction and in scientific language. In: Questions of philology, vol 1, pp. 35-47. Available online: http://www.infolex.ru/Concept.html

DESCLÉS, J.P. 1994. Réflexions sur les Grammaires Cognitives. In: Modèles Linguistiques. Available online: http://lalic.parissorbonne.fr/PUBLICATIONS/descles/modeles94.pdf

DHOUAILLY, C. 2019. Selfies, gloire et beauté: les stars millionnaires de la cosmétique. Available online: https://www.lemonde.fr/m-lemag/article/2019/04/19/selfies-gloire-et-beaute-les-stars-millionnaires-de-lacosmetique_5452557_4500055.html ISSN: 0395-2037

DICTIONARY.COM. 2020. Business. Available online: http://dictionary.reference.com/brow-se/business

DOBROSKLONSKAYA, T.G. 2005. Questions of studying media texts: Research experience of the modern English media language. Moscow: URSS. ISBN 5-35401133-7

DUCROT, O. 1998. Logique, structure, énonciation. Paris: ed. de Minuit. ISBN 9782707313102

EDGARD-ROSA, C. 2020. Danièle Kapel-Marcovici, femme d'affaire et de combats. In: Marieclaire. Available online: https://www.marieclaire.fr/daniele-kapel-marcovicifondation-raja-femme-d-affaire-et-de-combats, 1289890.asp 
EGOROVA, E.V. - KOCHELAEVA, E.Ya. 2016. Characteristic features of massmedia political discourse. In: Collection of articles on the materials of the conference "Lingua Academica: The relevance of the problem of linguistics and linguodidactics" Ulyanovsk: UST, pp. 31-37.

EGOROVA, E.V. - KRASHENINNIKOVA, N.A. - OSETROVA, O.I. 2016. We will never be brothers": implementation of attempts to reformat the stereotype "Brothers-Slavs". In: Modern studies of social problems, vol. 2, vol. 26, pp. 150-156. ISSN 2077-1770

ELIZAROVA, G.V. 2005. Culture and teaching foreign languages. Saint-Petersburg: KARO. ISBN 5-89815-597-X

FAGOT, V. 2019. La patronne des Apple Store quitte la marque à la pomme. In: Le Monde. Available online: https://www.lemonde.fr/economie/article/2019/02/06/lapatronne-des-apple-store-quitte-la-marque-a-la-pomme_5420164_3234.html ISSN: 0395-2037

FAIRCLOUGH, N. 2003. Analyzing Discourse: Textual Analysis for Social Research. London: Routledge. ISBN-13: 978-0415258937; ISBN-10: 0415258936

FUCHS, C. 2008. Linguistique française et cognition. In: Durand J., Habert B., Laks B. (éds.) Congrès Mondial de Linguistique Française. Paris: Institut de Linguistique Française. Available online: https://doi.org/10.1051/cmlf08340 ISBN 978-2-75980358-3

GARNIER, J. 2019. Christine Nagel, le nez des parfums Hermès. Available online: https://www.lemonde.fr/economie/article/2019/03/20/christine-nagel-le-nez-desparfums-hermes_5438676_3234.html ISSN 0395-2037

GAVRILOV, A.V. - RYABTSEVA, N.A. 2000. Report. The main stages of the feminist movement of the XIX-XX centuries. Available online: https://www.km.ru/referats/5D6D2B496B594C57956E380FD0B2CD55.

HEILMAN, M.E. - MARTELL, R.F. 1986. Exposure to Successful Women: Antidote to Sex Discrimination in Applicant Screening Decisions? In: Organizational Behavior and Human Decision Process, vol. 37, pp. 376-90. Available online: https://doi.org/10.1016/0749-5978(86)90036-1 ISSN 0749-5978

HELGESEN, S. 1990. The Female Advantage: Women's Way of Leadership. New York: Doubleday. ISBN 0385248784.

ISACHENKO, O.K. 2005. Business woman of Russia as a socio-cultural phenomenon: abstract of PhD: Stavropol: SSU. Available online: https://www.dissercat.com/content/delovaya-zhenshchina-rossii-kak-sotsiokulturnoeyavlenie

KARASIK, V.I. 2004. Language circle: personality, concepts, discourse. Moscow: Gnosis. ISBN 5-7333-0143-0

KLEIBER, G. 1981. Problèmes de référence : Descriptions définies et noms propres. Paris: Klincksieck. ISBN 2252023724

KLUSHINA, N.I. 2012. Russian national style and its implementation in the texts of modern media. In: Stylistyka, vol. 21, pp. 19-34. ISSN 1230-2287

KOCH, S. - LUFT, R. - KRUSE, L. 2005. Women and leadership - 20 years later: A semantic connotation study. In: Social Science Information, vol. 44, n. 1, pp. 9-39. ISSN 0539-0184

KOKOVA, A.V. 2017. Sociolinguistic aspect of the conceptualization of a business woman in the Russian language picture of the world. In: Actual problems of philology and pedagogical linguistics, vol. 2. pp. 107-111. Available online: https://cyberleninka.ru/article/n/sotsiolingvisticheskiy-aspekt-kontseptualizatsiidelovoy-zhenschiny-v-russkoy-yazykovoy-kartine-mira/viewer ISSN 2079-6021

KOZHEMYAKIN, E.A. 2010. Mass communication and media discourse: towards research methodology. In: Scientific Bulletin of the Belgorod State University. Series: Humanities, vol. 12, n. 83, pp. 13-21. Available online: 
https://cyberleninka.ru/article/n/massovaya-kommunikatsiya-i-mediadiskurs-kmetodologii-issledovaniya/viewer ISSN 2075-4574

KRASNYKH, V.V. 2002. Ethnopsycholinguistics and linguoculturology: a course of lectures. Moscow: Gnosis. ISBN 5-94244-009-3

KURKIMBAYEVA, A.M. - AKHATOVA B.A. - GUMOVSKAYA G.N. KOTENYATKINA I.B. - KHUZIAKHMETOV A.N. 2020. Communicative strategies in modern linguistics (on the material of English travel blogs). In: XLinguae, vol. 13, n. 2, pp. 33-49. Available online: https://doi.org/10.18355/XL.2020.13.02.03 ISSN 1337-8384, eISSN 2453-711X

LAZARD, G. 2007. La linguistique cognitive n'existe pas. In : Bulletin de la Société de linguistique de Paris, vol. 1. pp. 3-16. ISSN 0037-9069

LEEUWEN, T.V. 2008. Discourse and Practice. New tools for critical discourse analysis. New York: Oxford University Press. ISBN-13 978-0195323313; ISBN-10 0195323319

LE PARISIEN. 1998. Femme d'affaires. Available online: http://dictionnaire.sensagent.leparisien.fr/femme\%20d'affaires/fr-fr/

LYSAKOVA, I.P. 1989. Newspaper type and publication style: the experience of sociolinguistic research. Leningrad: LSU Publishing House. ISBN 5-288-00308-4

MASLOVA, V.A. 2011. Introduction to cognitive Linguistics. Moscow: FLINTA. ISBN 978-2-7598-0358-3

NOVIKOVA, N.V. 2009. Socio-philosophical analysis of the phenomenon of "business woman": PhD Thesis. Rostov-on-Don: Don State Technical University. Available online: https://www.dissercat.com/content/sotsialno-filosofskii-analizfenomena-delovaya-zhenshchina

PAVLOVSKAYA, A.V. 1998. Ethnic stereotypes in the light of intercultural communication. Moscow: Prosveshchenie.

PIMENOVA, M.V. 2011. Language picture of the world. Saint-Petersburg: St. Petersburg State University. ISBN 978-5-8154-02-24-9

POPOVA, Z.D. - STERNIN, I.A. 2010. Cognitive linguistics. Moscow: AST. ISBN 978-5-17-045103-6.

PRIVALOVA, I.V. 2004. The structure and the constituents of ethnolinguisticcultural consciousness. In: The Siberian Journal of Philology, n. 2, pp. 54-59. ISSN 1813-7083

PRIVALOVA, I.V. - SHAIDULLINA, A.R. - ZHELTUKHINA, M.R. GRINBERG, T.E. - GARCIA CASELLES, C. 2019. Coerced loss of national colorings - linguistic issues of virtual team communication. In: XLinguae, vol. 12, n.1, pp. 151-164. Available online: https://doi.org/10.18355/XL.2019.12.01.12 ISSN 1337-8384, eISSN 2453-711X

RASTIER, F. 1993. La sémantique cognitive : éléments d'histoire et d'épistémologie. In: Histoire Épistémologie Langage, vol. 15, n. 1, pp. 153-187. eISSN 1638-1580.

SEBE, L. 2020. Le style de Noholita avec Nasty Gal. Available online: https://www.magazine-avantages.fr/le-style-de-noholita-avec-nasty-gal,191756.asp

SOLGANIK, G.Ya. - KLUSHINA, N.I. - SMIRNOVA, N.V. 2014. Discourse and style theoretical and applied aspects. Moscow: FLINTA. ISBN 978-5-9765-1906-0

TAMERYAN, T.YU. - POPOVA, T.G. - REDKOZUBOVA, E.A. - ANIKEEVA, I.G. - SEDLYAROVA, O.M. - SOLOVIEVA, N.S. 2019. Feminine perspective of ethnic business communication. In: Espacios, vol. 40, n. 34, pp. 18-25. ISSN 07981015

UFIMTSEVA, N.V. 1995. Ethnic and cultural stereotypes: cross-cultural research. In: Bulletin of the Academy of Sciences. Literature and Language series. vol. 54, n. 3. pp. 55-62. ISSN 0321-1711

VASILYEVA, A.N. 1982. Newspaper-journalistic style of speech. Moscow: Russian language. FB Б 76-35/301 
VEPREVA, I.T. 2002. Language reflection in the post-Soviet era. Yekaterinburg: Ural Publishing House. ISBN 5-7584-0094-7

VIANSSON-PONTÉ, P. 2019. Simone de Beauvoir au «Monde», en 1978 : «J'ai cru trop vite à une proche victoire des femmes». Available online: https://www.lemonde.fr/idees/article/2019/03/08/simone-de-beauvoir-au-monde-en-

1978-j-ai-cru-trop-vite-a-une-proche-victoire-des-femmes_5432979_3232.html ISSN 0395-2037

ZHELTUKHINA, M.R. 2003. Tropological suggestiveness of mass-medial discourse and on the problem of speech influence of tropes in the language of mass media. Moscow: Institute of Linguistics of the Russian Academy of Sciences. ISBN 5-88234568-5

ZHELTUKHINA, M.R - KLUSHINA, N.I. - PONOMARENKO, E.B. VASILKOVA, N.N. - DZYUBENKO, A.I. 2017. Modern media influence: mass culture - mass consciousness - mass communication. In: XLinguae, vol. 1, n. 4, pp. 96-105. Available online: https://doi.org/10.18355/XL.2017.10.04.09 ISSN 13378384, eISSN 2453-711X

Words: 9289

Characters: 65299 (36,28 standard pages)

Lecturer Raigul Muratovna Baktiyarova

Departement of Kazakh Language and Literature Named after Academician S.S. Kirabaev

Kazakh National Pedagogical University Named after Abay

Abylai khan Kazakh university of international relations and world languages 13 Dostyk Ave.

050010, Almaty

Republic of Kazakhstan

rai_bah@mail.ru

Prof. Kalbike Umirbaevna Yessenova, DrSc.

Departement of Kazakh Language and Literature Named after Academician S.S. Kirabaev

Kazakh National Pedagogical University Named after Abay

13 Dostyk Ave.

050010, Almaty

Republic of Kazakhstan

kalbike_65@mail.ru

Prof. Marina Rostislavovna Zheltukhina, DrSc.

Institute of Foreign Languages

Volgograd State Socio-Pedagogical University, Moscow City University

27 Lenin Ave.

400066 Volgograd

4-1 2nd Selskohoziajstvenny proezd

129226 Moscow

Russia

zheltukhinamr@mgpu.ru

Prof. Irina Vladimirovna Privalova, DrSc.

Leading Research Felllow at the Research Laboratory "Intellectual Technologies of Text Management"

Institute of Philology and Intercultural Communication

XLinguae, Volume 14 Issue 4, October 2021, ISSN 1337-8384, eISSN 2453-711X 
Kazan (Volga region) Federal University

2-33 Tatarstan Street

42002 Republic of Tatarstan, Kazan

Russia

ivprivalova@mail.ru

Ass. Prof. Elena Borisovna Ponomarenko, PhD

Department of Foreign Languages

Peoples' Friendship University of Russia (RUDN University)

6 Miklukho-Maklaya Street

117198 Moscow

Russia

elepono@mail.ru 